\title{
High Accuracy Fundamental Matrix Computation and Its Performance Evaluation
}

\author{
Kenichi Kanatani \\ Department of Computer Science, Okayama University, \\ Okayama 700-8530 Japan \\ kanatani@suri.it.okayama-u.ac.jp \\ Yasuyuki Sugaya \\ Department of Information and Computer Sciences, \\ Toyohashi University of Technology, Toyohashi, Aichi 441-8580 Japan \\ sugaya@iim.ics.tut.ac.jp
}

\begin{abstract}
We compare the convergence performance of different numerical schemes for computing the fundamental matrix from point correspondences over two images. First, we state the problem and the associated KCR lower bound. Then, we describe the algorithms of three well-known methods: FNS, HEIV, and renormalization, to which we add Gauss-Newton iterations. For initial values, we test random choice, least squares, and Taubin's method. Experiments using simulated and real images reveal different characteristics of each method. Overall, FNS exhibits the best convergence performance.
\end{abstract}

\section{Introduction}

Computing the fundamental matrix from point correspondences over two images is the first step of many vision applications including camera calibration, image rectification, structure from motion, and new view generation. Well known numerical schemes for optimal fundamental matrix estimation in the presence of noise are FNS [2] and HEIV [11], which compute maximum likelihood (ML) in different ways. The solution is optimal in the sense that its covariance matrix agrees with the theoretical accuracy bound (KCR lower bound) except for higher order terms in noise [1, 8]. Kanatani's renormalization [8] is also known to be nearly equivalent to FNS and HEIV [9]. In this paper, we add a fourth method: directly computing ML by Gauss-Newton iterations.

All these are iterative methods with different convergence properties, which also depend on the choice of initial values. The purpose of this paper is to experimentally compare their convergence performance.

In Sec. 2, we state the problem and the KCR lower bound. In Sec. 3, we describe the four algorithms: FNS, HEIV, renormalization, and Gauss-Newton iterations. In Sec. 4, we introduce three types of initialization: random choice, least squares, and Taubin's method. Sec. 5 shows numerical examples using simulated and real images, together with discussions about the origins of the performance difference among them. In Sec. 6, we conclude that overall FNS has the best convergence properties. 


\section{Fundamental Matrix Estimation}

If point $(x, y)$ corresponds to point $\left(x^{\prime}, y^{\prime}\right)$ over two images of the same scene, we have the following epipolar equation [6]:

$$
\left(\left(\begin{array}{c}
x \\
y \\
f_{0}
\end{array}\right),\left(\begin{array}{lll}
F_{11} & F_{12} & F_{13} \\
F_{21} & F_{22} & F_{23} \\
F_{31} & F_{32} & F_{33}
\end{array}\right)\left(\begin{array}{c}
x^{\prime} \\
y^{\prime} \\
f_{0}
\end{array}\right)\right)=0 .
$$

Throughout this paper, we denote the inner product of vectors $\mathbf{a}$ and $\mathbf{b}$ by $(\mathbf{a}, \mathbf{b})$. In eq. (1), the matrix $\mathbf{F}=\left(F_{i j}\right)$ is called the fundamental matrix, where $f_{0}$ is an appropriate scale constant for stabilizing numerical computation [5]. If we define vectors

$$
\begin{aligned}
& \mathbf{u}=\left(F_{11}, F_{12}, F_{13}, F_{21}, F_{22}, F_{23}, F_{31}, F_{32}, F_{33}\right)^{\top}, \\
& \xi=\left(x x^{\prime}, x y^{\prime}, x f_{0}, y x^{\prime}, y y^{\prime}, y f_{0}, f_{0} x^{\prime}, f_{0} y^{\prime}, f_{0}^{2}\right)^{\top},
\end{aligned}
$$

eq. (1) is written as

$$
(\mathbf{u}, \xi)=0 \text {. }
$$

The absolute scale of the vector $\mathbf{u}$ is indeterminate, so we adopt normalization $\|\mathbf{u}\|=1$.

Fundamental matrix estimation thus reduces to fitting a hyperplane of the form of eq. (3) to noisy vector data $\left\{\xi_{\alpha}\right\}$ in $\mathscr{R}^{9}$. In this paper, we assume that outliers have already been removed. Let us write

$$
\xi_{\alpha}=\bar{\xi}_{\alpha}+\Delta \xi_{\alpha}
$$

where $\bar{\xi}_{\alpha}$ is the noiseless value, and $\Delta \xi_{\alpha}$ the noise term. We define the covariance matrix of $\xi_{\alpha}$ by $V\left[\xi_{\alpha}\right]=E\left[\Delta \xi_{\alpha} \Delta \xi_{\alpha}^{\top}\right]$, where $E[\cdot]$ denotes expectation for the noise distribution.

If each image coordinate of matching points is perturbed by independent random noise of mean 0 and standard deviation $\sigma$, the covariance matrix $V\left[\xi_{\alpha}\right]$ has the form $\sigma^{2} V_{0}\left[\xi_{\alpha}\right]$ up to $O\left(\sigma^{4}\right)$, where

$$
V_{0}\left[\xi_{\alpha}\right]=\left(\begin{array}{ccccccccc}
\bar{x}_{\alpha}^{2}+\bar{x}_{\alpha}^{\prime 2} & \bar{x}_{\alpha}^{\prime} \bar{y}_{\alpha}^{\prime} & f_{0} \bar{x}_{\alpha}^{\prime} & \bar{x}_{\alpha} \bar{y}_{\alpha} & 0 & 0 & f_{0} \bar{x}_{\alpha} & 0 & 0 \\
\bar{x}_{\alpha}^{\prime} \bar{y}_{\alpha}^{\prime} & \bar{x}_{\alpha}^{2}+\bar{y}_{\alpha}^{\prime 2} & f_{0} \bar{y}_{\alpha}^{\prime} & 0 & \bar{x}_{\alpha} \bar{y}_{\alpha} & 0 & 0 & f_{0} \bar{x}_{\alpha} & 0 \\
f_{0} \bar{x}_{\alpha}^{\prime} & f_{0} \bar{y}_{\alpha}^{\prime} & f_{0}^{2} & 0 & 0 & 0 & 0 & 0 & 0 \\
\bar{x}_{\alpha} \bar{y}_{\alpha} & 0 & 0 & \bar{y}_{\alpha}^{2}+\bar{x}_{\alpha}^{\prime 2} & \bar{x}_{\alpha}^{\prime} \bar{y}_{\alpha}^{\prime} & f_{0} \bar{x}_{\alpha}^{\prime} & f_{0} \bar{y}_{\alpha} & 0 & 0 \\
0 & \bar{x}_{\alpha} \bar{y}_{\alpha} & 0 & \bar{x}_{\alpha}^{\prime} \bar{y}_{\alpha}^{\prime} & \bar{y}_{\alpha}^{2}+\bar{y}_{\alpha}^{\prime 2} & f_{0} \bar{y}_{\alpha}^{\prime} & 0 & f_{0} \bar{y}_{\alpha} & 0 \\
0 & 0 & 0 & f_{0} \bar{x}_{\alpha}^{\prime} & f_{0} \bar{y}_{\alpha}^{\prime} & f_{0}^{2} & 0 & 0 & 0 \\
f_{0} \bar{x}_{\alpha} & 0 & 0 & f_{0} \bar{y}_{\alpha} & 0 & 0 & f_{0}^{2} & 0 & 0 \\
0 & f_{0} \bar{x}_{\alpha} & 0 & 0 & f_{0} \bar{y}_{\alpha} & 0 & 0 & f_{0}^{2} & 0 \\
0 & 0 & 0 & 0 & 0 & 0 & 0 & 0 & 0
\end{array}\right) .
$$

Here, $\left(\bar{x}_{\alpha}, \bar{y}_{\alpha}\right)$ and $\left(\bar{x}_{\alpha}^{\prime}, \bar{y}_{\alpha}^{\prime}\right)$ are the true positions of $\left(x_{\alpha}, y_{\alpha}\right)$ and $\left(x_{\alpha}^{\prime}, y_{\alpha}^{\prime}\right)$, respectively. They are replaced by $\left(x_{\alpha}, y_{\alpha}\right)$ and $\left(x_{\alpha}^{\prime}, y_{\alpha}^{\prime}\right)$ in actual computation ${ }^{1}$.

Let $\hat{\mathbf{u}}$ be an estimate of $\mathbf{u}$. We define its covariance matrix $V[\hat{\mathbf{u}}]$ by

$$
V[\hat{\mathbf{u}}]=E\left[\left(\mathbf{P}_{\mathbf{u}} \hat{\mathbf{u}}\right)\left(\mathbf{P}_{\mathbf{u}} \hat{\mathbf{u}}\right)^{\top}\right]
$$

\footnotetext{
${ }^{1}$ It has been confirmed by simulation that this replacement or omission of terms $O\left(\sigma^{4}\right)$ does not produce any significant changes.
} 
where $\mathbf{P}_{\mathbf{u}}$ is the following projection matrix (I denotes the unit matrix):

$$
\mathbf{P}_{\mathbf{u}}=\mathbf{I}-\mathbf{u u}^{\top}
$$

Since $\mathbf{u}$ is normalized to unit norm, its domain is the unit sphere $\mathscr{S}^{8}$ in $\mathscr{R}^{9}$. Eq. (6) means that the error is evaluated after projected onto the tangent space to $\mathscr{S}^{8}$ at $\mathbf{u}$.

It has been shown by Kanatani [8] that if $\xi_{\alpha}$ is identified with an independent Gaussian random variable of mean $\bar{\xi}_{\alpha}$ and covariance matrix $V\left[\xi_{\alpha}\right]$, the covariance matrix $V[\hat{\mathbf{u}}]$ of any unbiased estimator satisfies

$$
V[\hat{\mathbf{u}}] \succ \sigma^{2}\left(\sum_{\alpha=1}^{N} \frac{\bar{\xi}_{\alpha} \bar{\xi}_{\alpha}^{\top}}{\left(\mathbf{u}, V_{0}\left[\xi_{\alpha}\right] \mathbf{u}\right)}\right)^{-}
$$

where the relation $\succ$ means that the left-hand minus the right is positive semidefinite. The superscript - denotes pseudoinverse (of rank 8).

Chernov and Lesort [1] called the right-hand side of eq. (8) the KCR (KanataniCramer-Rao) lower bound and showed that this holds except for $O\left(\sigma^{4}\right)$ even if $\hat{\mathbf{u}}$ is not unbiased; it is sufficient that $\hat{\mathbf{u}}$ is "consistent" in the sense that $\hat{\mathbf{u}} \rightarrow \mathbf{u}$ as $\sigma \rightarrow 0$.

\section{Maximum Likelihood Estimation}

If $\xi_{\alpha}$ is regarded as an independent Gaussian random variable of mean $\bar{\xi}_{\alpha}$ and covariance matrix $V\left[\xi_{\alpha}\right]$, maximum likelihood $(M L)$ estimation is to minimize the sum of the square Mahalanobis distances of the data points $\xi_{\alpha}$ to the hyperplane to be fitted in $\mathscr{R}^{9}$, minimizing

$$
J_{\mathrm{ML}}=\frac{1}{2} \sum_{\alpha=1}^{N}\left(\xi_{\alpha}-\bar{\xi}_{\alpha}, V_{0}\left[\xi_{\alpha}\right]^{-}\left(\xi_{\alpha}-\bar{\xi}_{\alpha}\right)\right),
$$

subject to the constraint $\left(\mathbf{u}, \bar{\xi}_{\alpha}\right)=0, \alpha=1, \ldots, N$, where we can use $V_{0}\left[\xi_{\alpha}\right]$ instead of $V\left[\xi_{\alpha}\right]$ because the solution is unchanged if $V_{0}\left[\xi_{\alpha}\right]$ is multiplied by a positive constant. Introducing Lagrange multipliers for the constraint $\left(\mathbf{u}, \bar{\xi}_{\alpha}\right)=0$, we can reduce the problem to unconstrained minimization of the following function $[2,8,11]$ :

$$
J_{\mathrm{ML}}=\frac{1}{2} \sum_{\alpha=1}^{N} \frac{\left(\mathbf{u}, \xi_{\alpha}\right)^{2}}{\left(\mathbf{u}, V_{0}\left[\xi_{\alpha}\right] \mathbf{u}\right)}
$$

The solution is obtained by solving

$$
\nabla_{\mathbf{u}} J_{\mathrm{ML}}=\sum_{\alpha=1}^{N} \frac{\left(\mathbf{u}, \xi_{\alpha}\right) \xi_{\alpha}}{\left(\mathbf{u}, V_{0}\left[\xi_{\alpha}\right] \mathbf{u}\right)}-\sum_{\alpha=1}^{N} \frac{\left(\mathbf{u}, \xi_{\alpha}\right)^{2} V_{0}\left[\xi_{\alpha}\right] \mathbf{u}}{\left(\mathbf{u}, V_{0}\left[\xi_{\alpha}\right] \mathbf{u}\right)^{2}}=(\mathbf{M}-\mathbf{L}) \mathbf{u}=\mathbf{0}
$$

where we define

$$
\mathbf{M}=\sum_{\alpha=1}^{N} \frac{\xi_{\alpha} \xi_{\alpha}^{\top}}{\left(\mathbf{u}, V_{0}\left[\xi_{\alpha}\right] \mathbf{u}\right)}, \quad \mathbf{L}=\sum_{\alpha=1}^{N} \frac{\left(\mathbf{u}, \xi_{\alpha}\right)^{2} V_{0}\left[\xi_{\alpha}\right]}{\left(\mathbf{u}, V_{0}\left[\xi_{\alpha}\right] \mathbf{u}\right)^{2}}
$$

We need not consider the normalization constraint $\|\mathbf{u}\|=1$, because eq. (10) is a homogeneous expression of degree 0 in $\mathbf{u}$. In fact, multiplication of $\mathbf{u}$ by a nonzero constant 
does not affect the value of $J_{\mathrm{ML}}$. Hence, the gradient $\nabla_{\mathbf{u}} J_{\mathrm{ML}}$ is always orthogonal to $\mathbf{u}$. It can be shown that the covariance matrix of the resulting solution $\hat{\mathbf{u}}$ coincides with the the KCR lower bound (the right-hand side of eq. (8) except for $O\left(\sigma^{4}\right)[1,8,9]$.

The fundamental matrix $\mathbf{F}$ should also satisfy the constraint $\operatorname{det} \mathbf{F}=0$ [5]. However, once the solution $\hat{\mathbf{u}}$ of eq. (11) is obtained, it can be easily corrected so as to satisfy $\operatorname{det} \mathbf{F}=$ 0 in such a way that the accuracy is equivalent to the constrained minimization of eq. (10) subject to $\operatorname{det} \mathbf{F}=0$ except for higher order terms in $\sigma$ [10]. So, we focus here on the solution of eq. (11).

\section{FNS}

The procedure called FNS (fundamental numerical scheme) of Chojnacki et al. [2] for solving eq. (11) is described as follows:

1. Initialize $\mathbf{u}$.

2. Compute the matrices $\mathbf{M}$ and $\mathbf{L}$ in eqs. (12).

3. Solve the eigenvalue problem

$$
(\mathbf{M}-\mathbf{L}) \mathbf{u}^{\prime}=\lambda \mathbf{u}^{\prime}
$$

and compute the unit eigenvector $\mathbf{u}^{\prime}$ for the eigenvalue $\lambda$ closest to 0 .

4. If $\mathbf{u}^{\prime} \approx \mathbf{u}$ except for sign, return $\mathbf{u}$ and stop. Else, let $\mathbf{u} \leftarrow \mathbf{u}^{\prime}$ and go back to Step 2 . Chojnacki et al. [3] also showed how to incorporate the constraint $\operatorname{det} \mathbf{F}=0$ in the above iterations. Later, they pointed out that convergence performance improves if we choose in Step 3 not the eigenvalue closest to 0 but the smallest one [4]. We call the above procedure the original FNS and the one using the smallest eigenvalue the modified FNS.

Whichever eigenvalue is chosen for $\lambda$, we have $\lambda=0$ after convergence. In fact, convergence means

$$
(\mathbf{M}-\mathbf{L}) \mathbf{u}=\lambda \mathbf{u}
$$

for some $\mathbf{u}$. Computing the inner product with $\mathbf{u}$ on both sides, we have

$$
(\mathbf{u}, \mathbf{M u})-(\mathbf{u}, \mathbf{L u})=\lambda .
$$

On the other hand, eqs. (12) imply that $(\mathbf{u}, \mathbf{M u})=(\mathbf{u}, \mathbf{L u})$ identically, so $\lambda=0$.

\section{HEIV}

Eq. (11) can be rewritten as

$$
\mathbf{M u}=\mathbf{L u} .
$$

The HEIV (heteroscedastic errors-in-variables) method of Leedan and Meer [11] is to iteratively solve the generalized eigenvalue problem $\mathbf{M u}=\lambda \mathbf{L u}$. However, we cannot directly solve this, because $\mathbf{L}$ is not positive definite. So, we write

$$
\xi_{\alpha}=\left(\begin{array}{c}
\mathbf{z}_{\alpha} \\
f_{0}^{2}
\end{array}\right), \quad \mathbf{u}=\left(\begin{array}{c}
\mathbf{v} \\
F_{33}
\end{array}\right), \quad V_{0}\left[\xi_{\alpha}\right]=\left(\begin{array}{cc}
V_{0}\left[\mathbf{z}_{\alpha}\right] & \mathbf{0} \\
\mathbf{0} & 0
\end{array}\right),
$$

and define $8 \times 8$ matrices $\tilde{\mathbf{M}}$ and $\tilde{\mathbf{L}}$ by

$$
\tilde{\mathbf{M}}=\sum_{\alpha=1}^{N} \frac{\tilde{\mathbf{z}}_{\alpha} \tilde{\mathbf{z}}_{\alpha}^{\top}}{\left(\mathbf{v}, V_{0}\left[\mathbf{z}_{\alpha}\right] \mathbf{v}\right)}, \quad \tilde{\mathbf{L}}=\sum_{\alpha=1}^{N} \frac{\left(\mathbf{v}, \tilde{\mathbf{z}}_{\alpha}\right)^{2} V_{0}\left[\mathbf{z}_{\alpha}\right]}{\left(\mathbf{v}, V_{0}\left[\mathbf{z}_{\alpha}\right] \mathbf{v}\right)^{2}}
$$


where we put

$$
\tilde{\mathbf{z}}_{\alpha}=\mathbf{z}_{\alpha}-\overline{\mathbf{z}}, \quad \overline{\mathbf{z}}=\sum_{\alpha=1}^{N} \frac{\mathbf{z}_{\alpha}}{\left(\mathbf{v}, V_{0}\left[\mathbf{z}_{\alpha}\right] \mathbf{v}\right)} / \sum_{\beta=1}^{N} \frac{1}{\left(\mathbf{v}, V_{0}\left[\mathbf{z}_{\beta}\right] \mathbf{v}\right)} .
$$

Then, eq. (16) is decomposed into the following two equations [4, 11]:

$$
\tilde{\mathbf{M}} \mathbf{v}=\tilde{\mathbf{L}} \mathbf{v}, \quad(\mathbf{v}, \overline{\mathbf{z}})+f_{0}^{2} F_{33}=0 .
$$

If we compute a 8-D unit vector $\mathbf{v}$ that satisfies the first equation, the second equation gives $F_{33}$, and $\mathbf{u}$ is given by

$$
\mathbf{u}=N\left[\left(\begin{array}{c}
\mathbf{v} \\
F_{33}
\end{array}\right)\right],
$$

where $N[\cdot]$ denotes normalization to unit norm. The vector $\mathbf{u}$ that satisfies the first of eqs. (20) is computed by the following iterations $[4,11]$ :

1. Initialize $\mathbf{v}$.

2. Compute the matrices $\tilde{\mathbf{M}}$ and $\tilde{\mathbf{L}}$ in eqs. (18).

3. Solve the generalized eigenvalue problem

$$
\tilde{\mathbf{M}} \mathbf{v}^{\prime}=\lambda \tilde{\mathbf{L}} \mathbf{v}^{\prime},
$$

and compute the unit eigenvector $\mathbf{v}^{\prime}$ for the eigenvalue $\lambda$ closest to 1 .

4. If $\mathbf{v}^{\prime} \approx \mathbf{v}$ except for sign, return $\mathbf{v}$ and stop. Else, let $\mathbf{v} \leftarrow \mathbf{v}^{\prime}$ and go back to Step 2 . However, Leedan and Meer [11] pointed out that choosing in Step 3 not the eigenvalue closest to 1 but the smallest one improves the convergence performance. We call the above procedure the original HEIV and the one using the smallest eigenvalue the modified HEIV.

Whichever eigenvalue is chosen for $\lambda$, we have $\lambda=1$ after convergence. In fact, convergence means

$$
\tilde{\mathbf{M}} \mathbf{v}=\lambda \tilde{\mathbf{L}} \mathbf{v}
$$

for some $\mathbf{v}$. Computing the inner product with $\mathbf{v}$ on both sides, we have

$$
(\mathbf{v}, \tilde{\mathbf{M}} \mathbf{v})=\lambda(\mathbf{v}, \tilde{\mathbf{L}} \mathbf{v}) .
$$

On the other hand, eqs. (18) imply that $(\mathbf{v}, \tilde{\mathbf{M}} \mathbf{v})=(\mathbf{v}, \tilde{\mathbf{L}} \mathbf{v})$ identically, so $\lambda=1$.

\section{Renormalization}

The renormalization of Kanatani [8] is to approximate the matrix $\mathbf{L}$ in eqs. (12) in the form

$$
\mathbf{L} \approx c \mathbf{N}, \quad \mathbf{N}=\sum_{\alpha=1}^{N} \frac{V_{0}\left[\xi_{\alpha}\right]}{\left(\mathbf{u}, V_{0}\left[\xi_{\alpha}\right] \mathbf{u}\right)} .
$$

The constant $c$ is determined so that $\mathbf{M}-c \mathbf{N}$ has eigenvalue 0 . This is done by the following iterations [8]:

1. Initialize $\mathbf{u}$ and let $c=0$.

2. Compute the matrix $\mathbf{M}$ in eqs. (12) and the matrix $\mathbf{N}$ in eqs. (25). 
3. Solve the eigenvalue problem

$$
(\mathbf{M}-c \mathbf{N}) \mathbf{u}^{\prime}=\lambda \mathbf{u}^{\prime}
$$

and compute the unit eigenvector $\mathbf{u}^{\prime}$ for the eigenvalue $\lambda$ closest to 0 .

4. If $\lambda \approx 0$, return $\mathbf{u}$ and stop. Else, let

$$
c \leftarrow c+\frac{\lambda}{\left(\mathbf{u}^{\prime}, \mathbf{N} \mathbf{u}^{\prime}\right)}, \quad \mathbf{u} \leftarrow \mathbf{u}^{\prime}
$$

and go back to Step 2.

It can be shown that the resulting solution has accuracy nearly equivalent to FNS and HEIV [9].

\section{Gauss-Newton Iterations (GN)}

Since the gradient $\nabla_{\mathbf{u}} J_{\mathrm{ML}}$ is given by eq. (11), the function $J_{\mathrm{ML}}$ in eq. (10) can be minimized by Newton iterations. If we evaluate the Hessian $\nabla_{\mathbf{u}}^{2} J_{\mathrm{ML}}$, the increment $\Delta \mathbf{u}$ in $\mathbf{u}$ is determined by solving

$$
\left(\nabla_{\mathbf{u}}^{2} J_{\mathrm{ML}}\right) \Delta \mathbf{u}=-\nabla_{\mathbf{u}} J_{\mathrm{ML}}
$$

Since $\nabla_{\mathbf{u}}^{2} J_{\mathrm{ML}}$ is singular (the function $J_{\mathrm{ML}}$ is constant in the direction of $\mathbf{u}$ ), the solution is indeterminate. However, if we use pseudoinverse and compute

$$
\Delta \mathbf{u}=-\left(\nabla_{\mathbf{u}}^{2} J_{\mathrm{ML}}\right)^{-} \nabla_{\mathbf{u}} J_{\mathrm{ML}},
$$

we obtain a unique solution, which is orthogonal to $\mathbf{u}$.

Differentiating eq. (11) and introducing Gauss-Newton approximation (i.e., ignoring terms that contain $\left(\mathbf{u}, \xi_{\alpha}\right)$ ), we see that the Hessian is nothing but the matrix $\mathbf{M}$ in eqs. (12). In order to compute pseudoinverse, we enforce $\mathbf{M}$, which is generally nonsingular, to have eigenvalue 0 for $\mathbf{u}$, using the projection matrix $\mathbf{P}_{\mathbf{u}}$ of eq. (7). The iteration procedure goes as follows:

1. Initialize $\mathbf{u}$.

2. Compute

$$
\mathbf{u}^{\prime}=N\left[\mathbf{u}-\left(\mathbf{P}_{\mathbf{u}} \mathbf{M} \mathbf{P}_{\mathbf{u}}\right)^{-}(\mathbf{M}-\mathbf{L}) \mathbf{u}\right] .
$$

3. If $\mathbf{u}^{\prime} \approx \mathbf{u}$, return $\mathbf{u}$ and stop. Else, let $\mathbf{u} \leftarrow \mathbf{u}^{\prime}$ and go back to Step 2 .

\section{Initialization}

We test the following three types of initialization to examine the dependence of convergence properties on initial values.

\section{Random Choice}

We generate nine independent Gaussian random numbers of mean 0 and standard deviation 1 and normalize the vector consisting of them into unit norm.

\section{Least Squares (LS)}


Approximating the denominators in eq. (10) by a constant, we minimize

$$
J_{\mathrm{LS}}=\frac{1}{2} \sum_{\alpha=1}^{N}\left(\mathbf{u}, \xi_{\alpha}\right)^{2}=\frac{1}{2}\left(\mathbf{u}, \mathbf{M}_{\mathrm{LS}} \mathbf{u}\right), \quad \mathbf{M}_{\mathrm{LS}}=\sum_{\alpha=1}^{N} \xi_{\alpha} \xi_{\alpha}^{\top} .
$$

The solution is obtained by the unit eigenvector of $\mathbf{M}_{\mathrm{LS}}$ for the smallest eigenvalue.

\section{Taubin's Method}

Replacing the denominators in eq. (10) by their average, we minimize the following function [12]:

$$
J_{\mathrm{TB}}=\frac{1}{2} \frac{\sum_{\alpha=1}^{N}\left(\mathbf{u}, \xi_{\alpha}\right)^{2}}{\sum_{\alpha=1}^{N}\left(\mathbf{u}, V_{0}\left[\xi_{\alpha}\right] \mathbf{u}\right)}=\frac{1}{2} \frac{\left(\mathbf{u}, \mathbf{M}_{\mathrm{LS}} \mathbf{u}\right)}{\left(\mathbf{u}, \mathbf{N}_{\mathrm{TB}} \mathbf{u}\right)}, \quad \mathbf{N}_{\mathrm{TB}}=\sum_{\alpha=1}^{N} V_{0}\left[\xi_{\alpha}\right] .
$$

The solution is obtained by solving the generalized eigenvalue problem

$$
\mathbf{M}_{\mathrm{LS}} \mathbf{u}=\lambda \mathbf{N}_{\mathrm{TB}} \mathbf{u}
$$

for the smallest eigenvalue. However, we cannot directly solve this, because $\mathbf{N}_{\mathrm{TB}}$ is not positive definite. So, we decompose $\xi_{\alpha}, \mathbf{u}$, and $V_{0}\left[\xi_{\alpha}\right]$ in the form of eqs. (17) and define $8 \times 8$ matrices $\tilde{\mathbf{M}}_{\mathrm{LS}}$ and $\tilde{\mathbf{N}}_{\mathrm{TB}}$ by

$$
\tilde{\mathbf{M}}_{\mathrm{LS}}=\sum_{\alpha=1}^{N} \tilde{\mathbf{z}}_{\alpha} \tilde{\mathbf{z}}_{\alpha}^{\top}, \quad \tilde{\mathbf{N}}_{\mathrm{TB}}=\sum_{\alpha=1}^{N} V_{0}\left[\mathbf{z}_{\alpha}\right],
$$

where

$$
\tilde{\mathbf{z}}_{\alpha}=\mathbf{z}_{\alpha}-\overline{\mathbf{z}}, \quad \overline{\mathbf{z}}=\frac{1}{N} \sum_{\alpha=1}^{N} \mathbf{z}_{\alpha}
$$

Then, eq. (33) is decomposed into two equations

$$
\tilde{\mathbf{M}}_{\mathrm{LS}} \mathbf{v}=\lambda \tilde{\mathbf{N}}_{\mathrm{TB}} \mathbf{v}, \quad(\mathbf{v}, \overline{\mathbf{z}})+f_{0}^{2} F_{33}=0 .
$$

If we compute the unit eigenvector $\mathbf{v}$ of the first equation for the smallest eigenvalue $\lambda$, the second equation gives $F_{33}$, and $\mathbf{u}$ is given in the form of eq. (21).

\section{Numerical Examples}

\section{Simulated Images}

Fig. 1(a) shows two simulated images of two planar grid planes joined at angle $60^{\circ}$. The image size is $600 \times 600$ (pixels), and the focal length is 1200 (pixels). We added random Gaussian noise of mean 0 and standard deviation $\sigma$ (pixels) to the image coordinates of each grid point independently and estimated the fundamental matrix by FNS, HEIV, renormalization, and Gauss-Newton iterations (GN).

Fig. 1(b) plots for each $\sigma$ the root-mean-squares of $\left\|\mathbf{P}_{\mathbf{u}} \hat{\mathbf{u}}\right\|$ over 1000 independent trials. We compared LS, Taubin's method, and the four iterative methods starting from the Taubin solution and confirmed that for each method the final solution does not depend on 

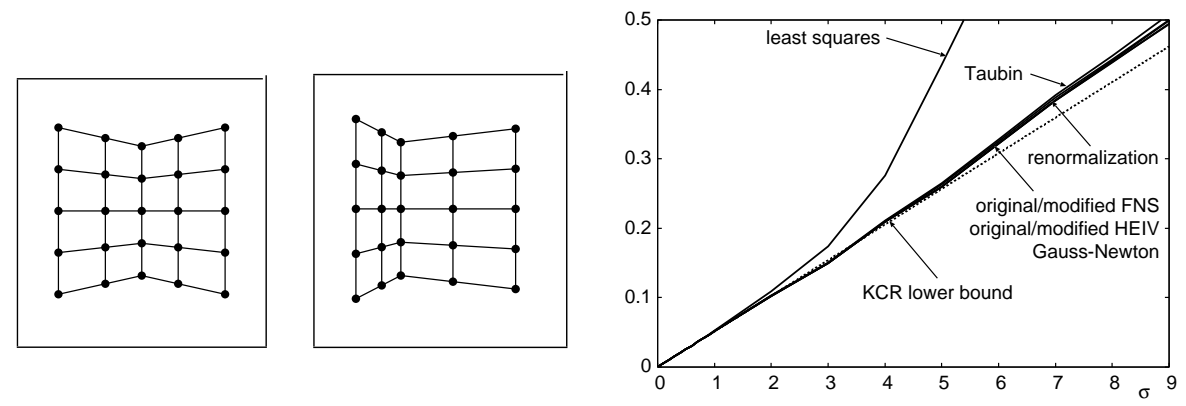

Figure 1: Left: Simulated images of planar grid surfaces. Right: Root-mean-squares error vs. noise level.

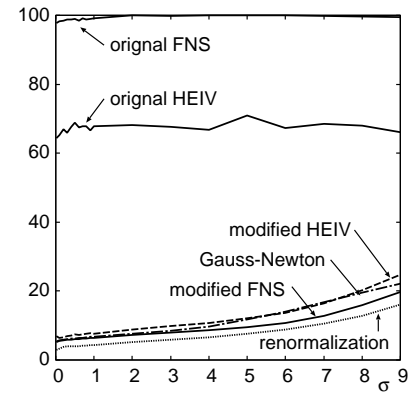

(a) Random initialization

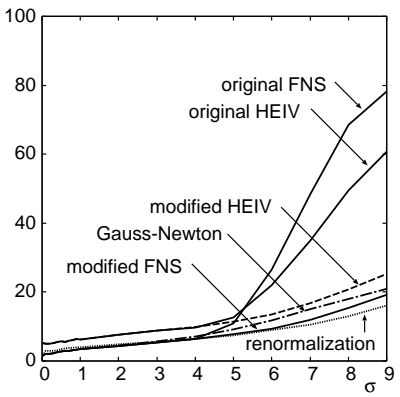

(b) LS initialization

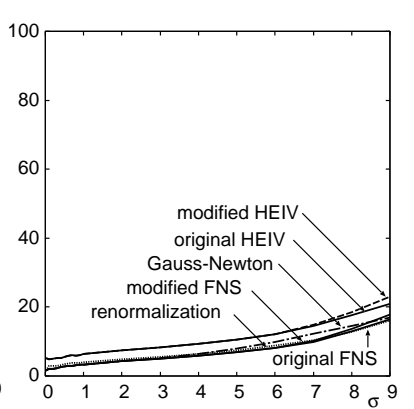

(a) Taubin initialization

Figure 2: Average number of iterations vs. noise level.

the initial value as long as the iterations converge. The dotted line indicates the KCR lower bound implied by Eq. (8). We can see that Taubin's method is considerably better than LS. The four iterative methods indeed improve the Taubin solution, but the improvement is rather small. All the solutions nearly agree with the KCR lower bound when noise is small and gradually deviate from it as noise increases. Since FNS, HEIV, and GN minimize the same function, the resulting solution is virtually the same. The renormalization solution is nearly equivalent to them.

Fig. 2 shows the average number of iterations of each method for 1000 trials. We stopped the iterations when the increment in $\mathbf{u}$ was less than $10^{-6}$ in norm. Fig. 2(a) is for random initialization. The original FNS did not converge for about $99 \%$ of the trials after 100 iterations; the original HEIV not for about 40\%. We stopped after 100 iterations and set the iteration count to 100 .

Fig. 2(a) shows that the modified FNS/HEIV converge much more quickly than the original FNS/HEIV. This can be explained as follows. If the computed $\mathbf{u}^{\prime}$ is close to the true value $\mathbf{u}$, the matrix $\mathbf{L}$ in eqs. (12) and the matrix $\tilde{\mathbf{L}}$ in eqs. (18) are both close to $\mathbf{O}$. Initially, however, they may be very different from $\mathbf{O}$, in particular when the initial value is randomly chosen. Eqs. (13) and (22) are written, respectively, as

$$
(\mathbf{M}-\mathbf{L}-\lambda \mathbf{I}) \mathbf{u}^{\prime}=\mathbf{0}, \quad(\tilde{\mathbf{M}}-\lambda \tilde{\mathbf{L}}) \mathbf{v}^{\prime}=\mathbf{0} .
$$



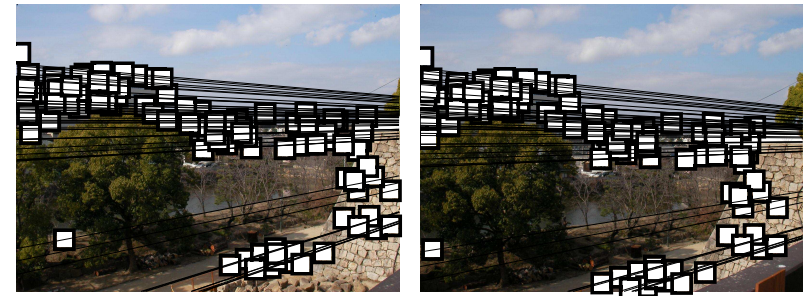

\begin{tabular}{c|r|r|r} 
& RD & LS & T \\
\hline o-FNS & 94.3 & 5 & 5 \\
m-FNS & 12.0 & 5 & 5 \\
o-HEIV & 74.6 & 7 & 7 \\
m-HEIV & 9.1 & 7 & 7 \\
renorm & 7.0 & 7 & 7 \\
GN & 10.3 & 5 & 6
\end{tabular}

Figure 3: Left: Corresponding points in real images and estimated epipolar lines. Right: Number of iterations of the original/modified FNS/HEIV (o/m-FNS/HEIV), renormalization (renorm) and GN for random initialization (RD), LS initialization (LS) and Taubin initialization (T).

The matrices $\mathbf{L}$ and $\tilde{\mathbf{L}}$ are both positive definite in general. In order that their effects be canceled, we need to choose $\lambda$ to be negative in the first equation and smaller than 1 in the second.

As predicted from this explanation, the difference between the original FNS/HEIV and the modified FNS/HEIV shrinks as we use better initial values, as seen from Fig. 2(b),(c). We also see that the (original or modified) FNS is more efficient than (original or modified) HEIV.

Another finding is that, for random initialization, renormalization is the most efficient. This is because we start solving eq. (26) with $c=0$, canceling the effect of $\mathbf{N}$ whatever it is initially, and the resulting $\mathbf{u}^{\prime}$ is close to the LS solution. In contrast, FNS and HEIV may produce a solution very different from the true value when initially the matrices $\mathbf{L}$ and $\tilde{\mathbf{L}}$ are very different from $\mathbf{O}$.

As Fig. 2(b),(c) shows, however, the convergence performance of FNS and HEIV improves as we use better initial values. Naturally, GN converges faster when started from better initial values. In contrast, renormalization behaves almost independently of initialization, confirming the above explanation. Overall, Taubin-initialized (original or modified) FNS shows the best convergence performance.

\section{Real Images}

Fig. 3 shows two images of the same scene on the left. We manually chose corresponding 100 points as marked there and computed the fundamental matrix by six different methods. The solution is the same whichever is used, and the estimated epipolar lines are drawn in the images.

The number of iterations for each method is listed on the right. For random initialization, we computed the average over 100 independent trials. We conclude that for whichever initialization, FNS is always better than HEIV. For both, the choice of the eigenvalue is irrelevant if the iterations are initialized by LS or Taubin's method; for random initialization, the original FNS/HEIV do not converge in most of the trials (recall that 100 means nonconvergence). As predicted, the number of iterations of renormalization does not depend on initialization. Overall, the LS or Taubin initialized (original or modified) FNS shows the best convergence performance. 


\section{Conclusions}

We have compared the convergence performance of different numerical schemes for computing the fundamental matrix from point correspondences over two images. First, we stated the problem and the associated KCR lower bound. Then, we described the algorithms of three well-known methods: FNS, HEIV, and renormalization, to which we added Gauss-Newton iterations (GN). For initial values, we tested random choice, LS, and Taubin's method. Experiments using simulated and real images revealed different characteristics of each method. Overall, FNS exhibited the best convergence performance.

Acknowledgments: This work was supported in part by the Ministry of Education, Culture, Sports, Science and Technology, Japan, under the Grant in Aid for Scientific Research C(2) (No. 15500113). The authors thank Wojciech Chojnacki of the University of Adelaide, Australia, and Nikolai Chernov of the University of Alabama at Birmingham, U.S.A. for helpful discussions.

\section{References}

[1] N. Chernov and C. Lesort, Statistical efficiency of curve fitting algorithms, Comput. Stat. Data Anal., 47-4 (2004-11), 713-728.

[2] W. Chojnacki, M. J. Brooks, A. van den Hengel and D. Gawley, On the fitting of surfaces to data with covariances, IEEE Trans. Patt. Anal. Mach. Intell., 22-11 (200011), 1294-1303.

[3] W. Chojnacki, M. J. Brooks, A. van den Hengel and D. Gawley, A new constrained parameter estimator for computer vision applications, Image Vision Comput., 22-2 (2004-2), 85-91.

[4] W. Chojnacki, M. J. Brooks, A. van den Hengel and D. Gawley, FNS, CFNS and HEIV: A unifying approach, J. Math. Imaging Vision, 23-2 (2005-9), 175-183.

[5] R. I. Hartley, In defense of the eight-point algorithm, IEEE Trans. Patt. Anal. Mach. Intell., 19-6 (1997-6), 580-593. oint

[6] R. Hartley and A. Zisserman, Multiple View Geometry in Computer Vision, Cambridge University Press, Cambridge, U.K., 2000.

[7] K. Kanatani, Geometric Computation for Machine Vision, Oxford University Press, Oxford, U.K., 1993.

[8] K. Kanatani, Statistical Optimization for Geometric Computation: Theory and Practice, Elsevier Science, Amsterdam, The Netherlands, 1996; Dover, New York, 2005.

[9] K. Kanatani, Further improving geometric fitting, Proc. 5th Int. Conf. 3-D Digital Imaging and Modeling, June, 2005, Ottawa, Ontario, Canada, pp. 2-13.

[10] K. Kanatani and N. Ohta, Comparing optimal three-dimensional reconstruction for finite motion and optical flow, J. Elec. Imaging, 12-3 (2003-7), 478-488.

[11] Y. Leedan and P. Meer, Heteroscedastic regression in computer vision: Problems with bilinear constraint, Int. J. Comput. Vision., 37-2 (2000-6), 127-150.

[12] G. Taubin, Estimation of planar curves, surfaces, and non-planar space curves defined by implicit equations with applications to edge and range image segmentation, IEEE Trans. Patt. Anal. Mach. Intell., 13-11 (1991-11), 1115-1138. 